\title{
Estilos de socialización en víctimas de acoso escolar
}

\author{
Ma Isabel Polo del Río, Benito León del Barco, Teresa Gómez Carroza, \\ Virginia Palacios García y Fernando Fajardo Bullón \\ Universidad de Extremadura (España)
}

\begin{abstract}
Un área de estudio importante en el acoso escolar continúa siendo el análisis de las causas del fenómeno y de los factores que actuarían como protección/riesgo en los ámbitos culturales, sociales, personales, escolares y familiares. La socialización puede ser clave como factor de protección o de riesgo en la aparición de situaciones de violencia escolar. Con esta investigación, mediante un análisis discriminante pretendemos analizar que variables de la socialización presentan un mayor poder de discriminación o cuantifican mejor las diferencias entre los distintos niveles de victimización en el acoso escolar. La muestra total estaba formada por 700 adolescentes estudiantes, $43 \%$ mujeres y $57 \%$ varones con una media de edad de 13.98 años. Hemos utilizado el Cuestionario de Convivencia Escolar (Defensor del Pueblo, 2006) y el BAS-3 (Batería de socialización, en formato de autoevaluación) de Silva y Martorell (1989), compuesta de 5 escalas de socialización: Consideración hacia los demás, autocontrol en las relaciones sociales, retraimiento social, ansiedad social/timidez, y liderazgo. Nuestros resultados confirman que niveles altos de victimización en el acoso escolar, estarían caracterizados por puntuaciones altas en ansiedad social/timidez y retraimiento social.
\end{abstract}

Palabras clave: Socialización, acoso escolar, víctima, adolescentes.

Socialization styles victims of bullying. An important area of study in bullying remains the analysis of the causes of the phenomenon and the factors that act as protective / risk in the cultural, social, personal, school and family. Socialization can be key protective factor or risk in the occurrence of school violence situations. With this research, using discriminant analysis we analyze that socialization variables have a higher discriminatory or better quantify the differences between the different levels of bullying victimization. The total sample consisted of 700 students adolescents, $43 \%$ males and $57 \%$ females with an average age of 13.98 years. We used the School Coexistence Questionnaire (Ombudsman, 2006) and BAS-3 (Battery socialization, self-assessment format) de Silva and Martorell (1989), consisting of 5 scales of socialization: Consideration for others, self in social relations, social withdrawal, social anxiety / shyness, and leadership. Our results confirm that high levels of victimization in bullying, would be characterized by high scores on social anxiety / shyness and social withdrawal.

Key words: Socialization, bullying, victims, adolescents.

Correspondencia: $\mathrm{M}^{\mathrm{a}}$ Isabel Polo del Río. Facultad de Formación del Profesorado. Universidad de Extremadura. Campus Universitario de Cáceres, s/n. C.P. 10071. Cáceres (España). E-mail: mabelpdrio@unex.es 
La socialización es un proceso a través del cual la persona aprende a interiorizar, en el transcurso de su vida, los elementos de su medio ambiente, los integra a la estructura de su personalidad y se adapta al entorno en el que vive (Rocher, 1993). Los vínculos afectivos que en la infancia establecemos con nuestros padres, hermanos o amigos, son unas de las bases más sólidas de nuestro desarrollo social.

La infancia y la adolescencia son periodos de la vida en los que la socialización primaria y las primeras etapas de la socialización secundaria son momentos especialmente importantes para su conformación social (Lahire, 2007). En la actualidad, niños y adolescentes están sometidos a múltiples agentes socializadores muy significativos para ellos: familia, grupo de iguales e institución escolar, cuya influencia es conjunta y, en ocasiones, contradictoria. Son los grupos de iguales, con quienes aprenden a conocerse a sí mismos, construyen su propia representación del mundo y adquieren las destrezas necesarias para integrarse en la sociedad; y es en las situaciones de aprendizaje escolar donde se generan con frecuencia dinámicas de agresión y victimización.

El bullying o maltrato escolar es una forma de agresión especialmente dañina y perjudicial (Trianes, Muñoz y Jiménez, 2007), y es definido como una "conducta de persecución física y/o psicológica que realiza un/a alumno/a contra otro/a, al que escoge como víctima de repetidos ataques" (Olweus, 1983). La problemática que genera va más allá de los episodios concretos de agresión y victimización, ya que cuando un sujeto recibe las agresiones de otro de manera sistemática, generaliza la percepción hostil al conjunto del ambiente escolar (Cerezo 2009), generando entre otros, graves estados de ansiedad y aislamiento social (Cerezo, 2002; Rigby, 2000). Según Ortega (1994), entre las consecuencias del acoso sobre la víctima están el destruir lentamente la autoestima y la confianza en sí misma, lo que provoca entre otros efectos una difícil adaptación social, llegando a estados depresivos o de ansiedad (León, 2009; Perren y Alsaker, 2006), y en situaciones extremas al suicidio.

Diversos estudios (Olweus, 1993; Schwartz, Dodge, Pettit y Bates, 1997; Salmivalli, Lagerspetz, Björkqvist, Österman, y Kauklainen, (1996); Pellegrini, Bartini, Brooks, 1999; Díaz-Aguado, Martínez y Martín, 2004; Smith, Talamelli, Cowie, Naylor, y Chauhan (2004) muestran dos perfiles de víctima. La víctima pasiva, se caracteriza por baja autoestima, ansiedad y depresión, tener pocos amigos y ser aislada socialmente por sus compañeros. Y por otro lado, la víctima activa, también aislada socialmente, con un matiz distinto en cuanto a sus relaciones sociales con el resto de compañeros: ser poco popular e incluso rechazada por ellos.

Un área de estudio importante en el acoso escolar continúa siendo el análisis de las causas del fenómeno y de los factores que actuarían como protección/riesgo en los ámbitos culturales, sociales, personales, escolares y familiares. La socialización puede ser clave como factor de protección o de riesgo en la aparición de situaciones de 
violencia escolar. Con esta investigación, mediante un análisis discriminante pretendemos analizar que variables de la socialización presentan un mayor poder de discriminación o cuantifican mejor las diferencias entre los distintos niveles de victimización en el acoso escolar.

\section{MÉTODO}

\section{Participantes}

La muestra total estaba formada por 700 adolescentes estudiantes, $43 \%$ mujeres y $57 \%$ varones con una media de edad de $13.98 \quad(D T=1.38)$. El número de participantes se determinó a partir del número de alumnos matriculados en Educación Secundaria Obligatoria (ESO) en centros públicos y concertados de la Comunidad de Extremadura durante el curso 2008-2009, considerando un error muestral de $3 \%$ y un nivel de confianza de $95.5 \%$. La selección de la muestra se realizó mediante un muestreo polietápico por conglomerados y selección aleatoria de los grupos en los centros que disponían de varias líneas en $\operatorname{los}$ cursos $1^{\circ}, 2^{\circ}, 3^{\circ}$ y $4^{\circ}$ de la ESO. El muestreo por conglomerados se llevó a cabo seleccionando al azar cuatro centros. En cuanto a la distribución por curso de nuestros participantes, 190 alumnos eran de $1^{\circ}$ de ESO, 177 de $2^{\circ}$ de ESO, 171 de $3^{\circ}$ y 162 de $4^{\circ}$ de ESO.

\section{Instrumentos}

Cuestionario sociodemográfico elaborado de forma específica para la investigación y que contenía cuestiones relativas a edad, género, nivel educativo y datos sociodemográficos y socioeconómicos de los padres.

Cuestionario de Convivencia Escolar (Defensor del Pueblo, 2007). Se utilizaron los resultados de tres ítems en los que se pedía que contestaran según una escala de tipo Likert de cuatro intervalos que van de 1 "nunca" a 4 "siempre", si habían vivido las 13 situaciones de acoso escolar desde la perspectiva de víctima, agresor/a y observador/a. Las situaciones son las siguientes: Ignorarle, no dejarle participar, insultarle, ponerle motes que le ofenden o ridiculizan, hablar mal de él o ella, esconderle cosas, romperle cosas, robarle, pegarle, amenazarle para meterle miedo, acosarle sexualmente, obligarle a hacer cosas que no quiere hacer con amenazas y amenazarle con armas.

El BAS-3 (Batería de socialización, en formato de autoevaluación) de Silva y Martorell (1989). Se compone de 5 escalas de socialización: 1. Consideración hacia los demás, con 14 elementos detecta sensibilidad social o preocupación por los demás, en particular por aquellos que tienen problemas y son rechazados. 2. Autocontrol en las relaciones sociales, se compone de 14 elementos recoge una dimensión que representa en su polo positivo acatamiento de normas y reglas sociales que facilitan la convivencia 
y el respeto, y en el polo negativo, conducta agresivas. 3. Retraimiento social, con 14 elementos detecta aislamiento de los demás. 4. Ansiedad social/timidez, con 12 elementos en las cuales se detecta manifestaciones de ansiedad unidas a reacciones de timidez. 5. Liderazgo, con 12 elementos, se detecta popularidad, confianza en sí mismo y espíritu de servicio.

En relación a la consistencia interna se encuentra en límites muy satisfactorios (Silva y Martorell, 1989), después de someter los datos a determinados análisis factoriales se llegó a una estructura final de 65 elementos y 5 escalas que se construyeron con los elementos de más alta saturación, escalas que hemos descrito anteriormente. La estabilidad temporal, según los autores, es asimismo satisfactoria, excepto para las escalas Consideración con los demás y Retraimiento social, las cuales deben tomarse con cautela para estudios longitudinales.

\section{Procedimiento}

Los datos que presentamos forman parte de una investigación más amplia relacionada con la convivencia escolar y bullying en centros de educación primaria y secundaria. Uno de los objetivos era conocer y analizar la relación entre conductas de victimización y variables de socialización.

El procedimiento seguido para la obtención de datos fue mediante la administración de los cuestionarios por grupo de clase. Previamente se había informado al centro y a los padres de los objetivos de la investigación y se había solicitado permiso al centro y los padres para su administración y a los participantes su consentimiento informado de forma verbal. Se aseguró a los participantes la confidencialidad de los datos obtenidos y su utilización exclusiva para fines de investigación. La administración de los cuestionarios ocupaba en torno a una hora y se llevó a cabo a lo largo de dos meses durante el tiempo de tutorías.

\section{RESULTADOS}

En primer lugar se seleccionaron diferentes subgrupos de la muestra final en función de los roles asumidos por los participantes. Para ello se utilizaron las puntuaciones en los tres ítems del Cuestionario del Defensor del Pueblo (2007) en los que se preguntaba a los sujetos por su participación, según los tres roles descritos por Olweus (1993), en diversas situaciones de bullying. En total son 13 situaciones distintas que se han enumerado en el apartado de instrumentos y a las que los sujetos debían responder en función de si habían sufrido (víctima), observado (observador) o provocado (agresor) esa situación. Para calcular la frecuencia de participación en esa situación se realizó un sumatorio de las respuestas a esos 13 ítems, de manera que obtuvimos dos nuevas variables denominadas intensidad de la experimentación de la situación de 
víctima y agresor. En estas variables se obtuvieron valores de entre 13 y 52, indicando el valor de 13, ausencia de experiencias y el valor 52 gran presencia de experiencias en todas las situaciones de bullying.

Una vez obtenidas estas variables se calcularon el valor de percentil 25 para seleccionar aquellos participantes que hubiesen experimentado un menor número e intensidad de las situaciones, y el valor de percentil 75 para seleccionar aquellos participantes que hubiesen experimentado un mayor número e intensidad de las situaciones. Las puntuaciones descriptivas de la variable víctima y los valores de los percentiles para cada uno de los niveles de intensidad fueron los siguientes: Víctimas $(M=14.53 ; D T=2.55 ;$ percentil $75=15$, percentil $25=13)$.

El análisis discriminante nos permite determinar la existencia de diferencias significativas entre los diferentes niveles de intensidad de victimización en el bullying en relación al conjunto de variables de Socialización. Igualmente, mediante el análisis discriminante pretendemos clasificar a los participantes y asignarlos a cada uno de los niveles de intensidad de victimización en el bullying en función del resultado de la combinación lineal del conjunto de variables independientes. En nuestro estudio hemos utilizado como variables independientes y predictoras las cinco escalas de socialización del BAS-3. Como variables dependientes los diferentes niveles de intensidad de victimización en el bullying.

En la tabla 1 podemos ver las medias y desviaciones típicas de cada uno de los niveles de intensidad de victimización en la dinámica bullying para las cinco escalas de Socialización. Los niveles de baja, media y alta intensidad de victimización obtienen puntuaciones medias más altas en Consideración hacia los demás y Autocontrol en las relaciones sociales.

Tabla 1. Medias y desviaciones típicas Escalas de Socialización del BAS-3 en función de los diferentes niveles de victimización del bullying

\begin{tabular}{lccccc}
\hline & \multicolumn{4}{c}{ Escala de Socialización BAS-3 } \\
\cline { 2 - 6 } & $\begin{array}{c}\text { Consideración } \\
\text { hacia los demás } \\
M(D T)\end{array}$ & $\begin{array}{c}\text { Autocontrol en las } \\
\text { relaciones sociales } \\
M(D T)\end{array}$ & $\begin{array}{c}\text { Retraimiento } \\
\text { Social } M(D T)\end{array}$ & $\begin{array}{c}\text { Ansiedad Social/ } \\
\text { Timidez } M(D T)\end{array}$ & $\begin{array}{c}\text { Liderazgo } \\
M(D T)\end{array}$ \\
\hline $\begin{array}{l}\text { Baja } \\
\text { Victimización }\end{array}$ & $25.62(3.25)$ & $24.72(2.95)$ & $15.65(3.39)$ & $15.33(3.29)$ & $18.32(2.51)$ \\
\hline $\begin{array}{l}\text { Media } \\
\text { Victimización }\end{array}$ & $26.19(3.14)$ & $24.77(4.33)$ & $16.02(3.32)$ & $16.04(2.74)$ & $18.43(2.98)$ \\
\hline $\begin{array}{l}\text { Alta } \\
\text { Victimización }\end{array}$ & $26.33(2.78)$ & $23.75(3.74)$ & $16.52(2.63)$ & $16.47(3.25)$ & $18.30(2.55)$ \\
\hline
\end{tabular}

A continuación, se examinó la posible existencia de diferencias entre las medias de los tres grupos de niveles de victimización en el bullying, en cuanto a las puntuaciones en las escalas de Socialización del BAS-3. Para ello se realizó un análisis de la varianza (ANOVA), encontrando diferencias significativas entre los tres niveles de victimización para dos de las escalas de socialización del BAS-3. Para la escala 
Autocontrol en las relaciones sociales (Wilks $\lambda=.988 ; F=5.343 ; p<.048$ ); Ansiedad Social/Timidez (Wilks $\lambda=.979 ; F=5.343 ; p<.005$ ).

Una vez demostradas la existencia de diferencias entre la medias de los tres niveles de victimización del bullying, analizaremos mediante el análisis discriminante, qué escalas de Socialización del BAS-3 explican en mayor medida esas diferencias. En la tabla 2 se muestra la matriz de estructura que se crea en el análisis discriminante. El número máximo de funciones discriminantes o combinaciones lineales es igual a una unidad menos que el número de grupos asignados a la variable dependiente. ¿Qué función tiene un mayor poder de discriminación y utilizaremos para interpretar los datos? El análisis de las funciones discriminantes nos indica que la Función 1 es la que presenta un mayor poder de discriminación entre los tres niveles de victimización.

Tabla 2. Matriz de la estructura. Variables ordenadas por el tamaño de la correlación con la función discriminante

\begin{tabular}{lcc}
\hline & \multicolumn{2}{c}{ Funciones } \\
\cline { 2 - 3 } & Función 1 & Función 2 \\
\hline Ansiedad Social/Timidez & $.616^{*}$ & .367 \\
\hline Retraimiento Social & $.441^{*}$ & -.062 \\
\hline Autocontrol en las relaciones sociales & -.386 & .882 \\
\hline Consideración hacia los demás & .395 & .503 \\
\hline Liderazgo & -.002 & .284 \\
\hline
\end{tabular}

Tabla 3. Resultados de la clasificación empleando la función discriminante

\begin{tabular}{lllll}
\hline & & \multicolumn{3}{c}{ Grupo de pertenencia pronosticado } \\
\cline { 2 - 5 } & & Baja victimización & Media victimización & Alta victimización \\
\hline \multirow{2}{*}{$\%$} & Baja victimización & 57.7 & 19.2 & 23.0 \\
\cline { 2 - 5 } & Media victimización & 38.3 & 23.4 & 38.3 \\
\cline { 2 - 5 } & Alta victimización & 33.0 & 16.0 & 50.9 \\
\hline
\end{tabular}

Nota: Clasificados correctamente el $44.1 \%$ de los casos agrupados originales.

La Función 1 explica un porcentaje de varianza muy superior al resto de funciones, muestra una mayor correlación canónica y distancia entre los grupos discriminados (Lambda de Wilks más cercano a 0). Además, el análisis de Chi cuadrado presenta el más elevado nivel de significación. Función 1 (\% de varianza=91.3; correlación canónica=.229; Wilks $\left.\lambda=.942 ; \chi^{2}=29.026, \mathrm{gl}=10 ; p<.001\right)$. Por tanto, según la Función 1 el factor que mayor capacidad discriminante tiene es Ansiedad Social/Timidez (.616). Le sigue el factor Retraimiento Social (.441).

Finalmente, en la tabla 3 observamos que la función canónica discriminante obtenida permite clasificar correctamente el $57.7 \%$ del nivel baja intensidad de victimización, el $23.4 \%$ del nivel media intensidad de victimización, el 50.9\% del nivel alta intensidad de victimización. Ganancias medias en la predicción superiores al 33\% que acertaríamos por azar en los 3 niveles de intensidad de victimización. 


\section{DISCUSIÓN}

El objetivo principal de este trabajo es analizar que variables de socialización presentan un mayor poder de discriminación o cuantifican mejor las diferencias entre los diferentes niveles de intensidad de victimización en el bullying. Los dos factores que mejor cuantifican, las diferencias entre los diferentes niveles de intensidad de victimización, son por este orden el factor Ansiedad Social/Timidez y el factor Retraimiento Social.

Estos resultados son coincidentes con las investigaciones realizadas por Cerezo (2001, 2002); Rigby (2000) concluyendo que la víctima del acoso escolar presenta altas tasas de ansiedad y timidez en sus relaciones, además de retraimiento social, lo que determina que los alumnos que sufren la situación de acoso escolar se encuentren en clara situación de indefensión en el grupo.

En este sentido, Moreno, Vacas y Roa (2006) concluyen que es el agresor, cuya acción negativa e intencionada, dirigida a desprestigiar socialmente a la víctima (Benítez y Justicia, 2006), provocan en ella un aislamiento con respecto a sus compañeros y una progresiva exclusión social (Griffin y Gross, 2004). La víctima comienza a percibir el entorno social como poco seguro y amenazante (Cerezo, 1997; Olweus, 1998; Sutton y Smith, 1999).

En sus investigaciones, Oñate y Piñuel (2005), concluyen por tanto, que el alumno víctima de acoso escolar, desarrolla fácilmente una introversión social que le aísla aún más de un entorno ya reducido significativamente, por la acción del propio agresor. Las consecuencias del acoso escolar dejan huellas imborrables en las víctimas, tanto a medio como a corto plazo, se sienten infelices, inseguros e incluso llegan a somatizar el alto nivel de ansiedad en el que viven, tal y como concluye Cerezo (2008).

En líneas de investigación futuras nos planteamos analizar que variables de la socialización presentan un mayor poder de discriminación entre los distintos niveles de agresión en el acoso escolar. Ciertos estudios tratan de establecer aspectos de socialización característicos de las figuras implicadas en la dinámica bullying (Cerezo, 2002).

Por otro lado, nos planteamos introducir otras variables en nuestro estudio, como son los estilos parentales y otras variables familiares, que puedan relacionarse con los comportamientos propios de la dinámica bullying. Estudios como los de Repetti, Taylor y Seeman (2002) confirman que estilos parentales caracterizados por niveles inadecuados de afecto, de apoyo y predominio de la agresión y el rechazo hacia los hijos, se relacionan con la manifestación de problemas conductuales de agresividad, hostilidad y delincuencia.

Finalmente, consideramos que es necesario implementar programas de prevención del acoso escolar en todos los centros educativos y coincidimos con 
Díaz-Aguado (2005) en la importancia de erradicar las situaciones de exclusión desde las primeras etapas educativas y favorecer la identificación de los adolescentes con los valores de respeto, empatía y no violencia, así como la necesidad de mantener una perspectiva ecológica y colaborativa entre las familias, centros educativos y recursos comunitarios.

\section{REFERENCIAS}

Benítez, J.L. y Justicia, F. (2006). El maltrato entre iguales: descripción y análisis del fenómeno. Revista de Investigación Psicoeducativa, 4(2), 81-93.

Cerezo, F. (1997). Conductas agresivas en la edad escolar. Madrid: Pirámide.

Cerezo, F. (2001) Variables de personalidad asociadas a la dinámica bullying (agresores versus víctimas) en niños y niñas de 10 a 15 años. Anales de Psicología, 17(1), 37-44.

Cerezo, F. (2002). El bullying y su relación con las actitudes de socialización en una muestra de adolescentes. Revista Electrónica Interuniversitaria de Formación del Profesorado, 5.

Cerezo, F. (2008). Acoso Escolar entre iguales. Efectos del bullying. Boletín de Pediatría, 48, 353 368.

Cerezo, F. (2009). Bullying: análisis de la situación en las aulas españolas. International Journal of Psychology and Psychological Therapy, 9(3), 367-378.

Defensor del Pueblo (2007). Violencia escolar: el maltrato entre iguales en la Educación Secundaria Obligatoria. Madrid: Publicaciones de la Oficina del Defensor del Pueblo.

Díaz-Aguado, M.J. (2005). La violencia entre iguales en la adolescencia y su prevención desde la escuela. Psicothema, 17, 549-558.

Díaz-Aguado, M.J., Martínez, R. y Martín, G. (2004) Prevención de la violencia y lucha contra la exclusión desde la adolescencia, 1. La violencia entre iguales en la escuela y en el ocio. Estudios comparativos e instrumentos de evaluación. Madrid: Instituto de la Juventud.

Griffin, R.S. y Gross, A.M. (2004). Childhood bullying: current empirical findings and future directions for research. Aggression and Violenta Behavior, 9, 379-400.

Lahire, B. (2007). Infancia y adolescencia: de los tiempos de socialización sometidos a constricciones múltiples. Revista de Antropología Social, 16, 21-38.

León, B. (2009). Salud mental en las aulas. Revista de estudios de juventud, 84, 66-83.

Moreno, M., Vacas, C. y Roa, J.M. (2006). Víctimización escolar y clima sociofamiliar. Revista Iberoamericana de Educación, 40(6), 12-17.

Olweus, D. (1983). Low school achievement and agressive behaviour in adolescent boys. En D. Magnusson y V. Allen (Eds.), Human developrnent. An interactional perspective (pp. 353-365). Nueva York: Academic Press.

Olweus, D. (1993). Bullying at school: What we know and what we can do. Cambridge, MA: Blackwell.

Olweus, D. (1998). Conductas de acoso y amenaza entre escolares. Madrid: Morata.

Oñate, A. y Piñuel, I. (2005). Informe Cisneros VII: Violencia y acoso escolar en alumnos de Primaria, ESO y Bachiller. Madrid: Instituto de Innovación educativa y Desarrollo directivo.

Ortega, R. (1994). Violencia interpersonal en los centros educativos de enseñanza secundaria. Un estudio sobre el maltrato e intimidación entre compañeros. Revista de Educación, 304, 253-280.

Pellegrini, A., Bartini, M. y Brooks, F. (1999). School Bullyes, Victims and Aggressive Victims. Journal of Educational Psychology, 91, 216-224. 
Perren, S. y Alsaker, F.D. (2006). Social Behaviour and Peer Relationships of Victims, Bullyvictims, and Bullies in Kindergarten. The Journal of Child Psychology and Psychiatry and Allied Disciplines, 47, 45-57.

Repetti, R.L., Taylor, S.E. y Seeman, T.E. (2002). Risky families: Family social environments and the mental and physical health of offspring. Psychological Bulletin, 128(2), 330-366.

Rigby, K. (2000). Effects of peer victimisation in schools and perceived social support on adolescent well-being. Journal of Adolescence, 23, 57-68.

Rocher, G. (1993). Introducción a la psicología general. Barcelona: Editorial Trillos.

Salmivalli, C., Lagerspetz, K., Bjorkqvist, K., Osterman, K. y Kaukiainen, A. (1996). Los actos de molestia o intimidación como proceso grupal: Roles participantes y sus relaciones con el estatus social dentro del grupo. Comportamiento agresivo. Aggressive Behavior, 22, 115.

Schwartz, D., Dodge, K., Pettit, G. y Bates, J. (1977). The Early Socialization of Aggressive Victims. Child Development, 68, 665-675.

Silva, F. y Martorell, M.C. (1989). BAS.3. Batería de Socialización. Autoevaluación. Madrid: TEA.

Smith, R., Talamelli, L., Cowie, H., Naylor, P. y Chauhan, P. (2004). Profiles of non-victims, Escaped Victims, Continuing Victims and new Victims of School Bullying. British Journal of Educational Psychology, 74, 565-581.

Sutton, J. y Smith, P.K. (1999). Social cognition and bullying: Social inadequancy or skilled manipulation? British J. of Developmental Psychology, 17(3), 435-450.

Trianes, M.V., Muñoz, A. y Jiménez, M. (2007). Las relaciones sociales en la infancia y la adolescencia y sus problemas. Madrid: Pirámide.

Recibido: 17 de febrero de 2013

Recepción Modificaciones: 8 de marzo de 2013

Aceptado: 12 de marzo de 2013 